\title{
The potentially therapeutic targets of pediatric anaplastic ependymoma by transcriptome profiling
}

\author{
Jingsheng WANG ${ }^{1}$, Chen SUN ${ }^{2, *}$ Minggang LIU ${ }^{1}$, Dongdong ZANG ${ }^{1}$, Chong WANG ${ }^{1}$, Qibin LIU ${ }^{1}$, Yuecheng LIU ${ }^{1}$, Qian CHEN CHE $^{1, *}$ \\ ${ }^{1}$ Neurosurgery Department, Shenzhen Children's Hospital, Shenzhen, Guangdong, China; ${ }^{2}$ Wuhan Children's Hospital (Wuhan Maternal and \\ Child Healthcare Hospital), Tongji Medical College, Huazhong University of Science and Technology, Wuhan, Hubei, China
}

${ }^{*}$ Correspondence: peaceandlove9527@126.com

"Contributed equally to this work.

Received May 29, 2020 / Accepted August 12, 2020

\begin{abstract}
Ependymoma (EPN) is a type of tumor that occurs in the central nervous system of children and adults. EPN produces resistance to chemotherapy, and there are no targeted drugs available as a proper cure. Therefore, the use of high-throughput sequencing technologies to elucidate pathogenic mechanisms is of prime importance to identify potential tumor target genes helpful for developing effective therapeutic approaches against EPN. With this objective, we used RNA-seq analysis to identify differentially expressed genes (DEGs) and pathways in 4 pairs of EPN tissues and adjacent tissues. In total, we found 5,445 differentially expressed genes. The synaptic vesicle cycle and extracellular matrix (ECM) receptor interaction pathways were highly enriched in the ependymoma group. Nine differentially expressed genes (SNAP25, GRM4, CELSR1, LAMA1, WNT5A, ROR2, CCND1, EPHB2, FOXJ1) were randomly verified by RT-qPCR, supporting the authenticity of our sequencing results. This study provides global gene information and some new potential biomarkers for the diagnosis and therapeutic targets of ependymoma.
\end{abstract}

Key words: ependymoma, RNA-seq, differentially expressed genes, signaling pathway

Ependymoma is a type of tumor in the central nervous system (CNS) that originates from the neuroectoderm. It is ranked as the third most common brain tumor in children, causing death in approximately $45 \%$ of affected individuals [1]. According to its progression and malignancy, ependymoma has been classified into three grades by the World Health Organization (WHO) [2]: myxopapillary ependymoma and subependymoma (grade I), ependymoma (grade II), ependymoma RELA fusion-positive (grade II or III), and anaplastic ependymoma (grade III). Ependymoma can invade the entire neural axis. In children, it has been found mainly in intracranial regions, while the spine is the most common site for adults. Surgery and radiotherapy are standard recommended treatments for ependymoma, while chemotherapy has not been indicated to be effective in most cases [3]. The clinical utility of histopathological classification is limited since the ependymoma RELA fusionpositive subtype is not distinguishable in this manner [4]. To improve risk stratification, the molecular classification will be an important part of future clinical trials of ependymoma. For most molecular groups, the gene driving factors are unknown, so it is important to find the gene targets for ependymoma. With the development of genomics, transcriptomics, and epigenomics, it is of great significance to identify the invasive genes and biomarkers of ependymoma by using these techniques. Lukashova et al. found that the overexpression of genes in highly proliferative tumors was associated with poor prognosis using cDNA microarrays and RT-PCR [5]. Fukuoka et al. identified the posterior fossa ependymoma subgroup with $100 \%$ specificity using the methylation status of 3 genes, CRIP1, DRD4, and LBX2. Whole-genome sequencing and RNA sequencing have been conducted and reported for supratentorial ependymomas, revealing $70 \%$ C11orf95-RELA fusions in supratentorial ependymomas. This fusion has been reported to drive oncogenic NF-kB signaling in supratentorial ependymoma [6]. The histology of anaplastic ependymoma is equivalent to the grade III of $\mathrm{WHO}$, and it originates from ependymal malignant glioma, especially in children, grows rapidly, and has a poor clinical process. The special genetic changes that occur in ependymoma are not clear. In our research, we analyzed the transcriptome map between pediatric anaplastic ependymoma tissues and adjacent tissues and combined it with cBioPortal database profiling. Our findings may provide 
some clues about the pathogenesis of pediatric anaplastic ependymoma and may provide some new biomarkers for this disease.

\section{Patients and methods}

Patient sample collection. Anaplastic ependymoma tissues and adjacent tissues were collected from 4 ependymoma patients (11 months to 4 years old). Informed consent from the parents of these children was obtained from Shenzhen Children's Hospital. The clinical specimens included four ependymoma tissues and four adjacent tissues. None of the four patients received chemotherapy or radiotherapy. The ependymoma tissues were obtained from gray tissues, approximately $1 \mathrm{~cm}^{3}$ in size. The adjacent tissues were taken at a distance of at least $1 \mathrm{~cm}$ from the tumor, and there were no obvious tumor cells. The clinical specimens were immediately frozen in liquid nitrogen and then stored at $-80^{\circ} \mathrm{C}$. All tissues were diagnosed histologically. This study was approved by the Research Ethics Committee of the Shenzhen Children's Hospital. All ethical procedures conformed to the principles of the 1964 Declaration of Helsinki and its latest 2008 amendments. Since the patients were all minor children, we had obtained the written informed consent signed by the legal guardians of these participants.

RNA extraction and RNA-seq library construction. RNA was extracted from the ependymoma tissues and their respective normal/adjacent tissues. Then, mRNA-seq libraries were constructed by the VAHTS mRNA-seq V3 Library Prep Kit for Illumina (Vazyme, China) according to

Table 1. Primer Information.

\begin{tabular}{lll}
\hline Gene name & Primers $\mathbf{( 5}^{\prime}$----3') \\
\hline SNAP25 & forward & CAATGAGCTGGAGGAGATGCA \\
& reverse & TGCTTTCCAGCGACTCATCA \\
GRM4 & forward & TGAGGGTGCTGTCACGATCC \\
& reverse & ACGTGGCTGCCCTTCTTGAG \\
CELSR1 & forward & CGCTTCCACTTCACCATCTCCCT \\
& reverse & GCCACGGTCGTTGTTGTCTCG \\
LAMA1 & forward & GCCAGCTCTAATGCCATC \\
& reverse & GGGTTGACAAATTCCTCCAAA \\
Wnt5a & forward & CTTCGCCCAGGTTGT AATTGAAGC \\
& reverse & CTGCCAAAAAC AGAGGTGTTATCC \\
ROR2 & forward & GGATCCGAACGACCCTTTAG \\
& reverse & AGTAACCTTTCAGAGTTGGAATCG \\
CCND1 & forward & TATTGCGCTGCTACCGTTGA \\
& reverse & CCAATAGCAGCAAACAATGTGAAA \\
EphB2 & forward & TGAGTGCCCTCAGATGGTCAA \\
& reverse & AGGGCAGGGTATCACAGTGAATG \\
FOXJ1 & forward & CAGAATCGCTGCCTCCTCTC \\
& reverse & CAGGGTCCTTTAGCCGGTTT \\
GAPDH & forward & ACCACAGTCCATGCCATCAC \\
& reverse & TCCACCACCCTGTTGCTG TA \\
\hline
\end{tabular}

the protocol [6]. This step was followed by mRNA-seq on the Illumina HiSeq 2000 platform.

Bioinformatics analysis. The numbers of reads per kilobase of exon model per million mapped reads (RPKM) were calculated to obtain normalized gene expression levels. The FANSe2 alignment tool was used to map the original RNA-seq reads to the reference transcriptome (Human RefSeq-RNA hg19) [7]. Because of the high accuracy of the mapping algorithm, the genes obtained above 10 reads by sequencing were considered expressing genes, while the genes below 10 reads were considered not expressing [8]. We calculated the correlation coefficients to test the biological duplication efficiency, and principal component analysis was performed to show the different groups of the tumor and normal/adjacent samples. Next, we used edgeR for the analysis of the gene expression level, which was based on the negative binomial distribution $[9,10]$. To eliminate biological variation, the screening of DEGs was evaluated from two aspects: the fold change and the significance level. The screening threshold of DEGs in this analysis was set as $\mid \log 2$ (fold change) $\mid>1$ and FDR $<0.01$. A volcano plot was constructed to show the differentially expressed genes (DEGs). We performed hierarchical clustering to evaluate the expression levels of the DEGs in different samples.

Pathway analysis. Gene Ontology (GO) analysis of the DEGs was conducted by topGO software (version 2.18.0) and Fisher's exact test was used for statistical testing. The gene list ordered by $\log 2$ FoldChange from edgeR was submitted to GSEA using ClusterProfiler. The GO terms in ependymoma and peritumoral tissues with significant enrichment results were identified based on the normalized enrichment score (NES) and P value. The ClusterProfiler and DOSE R packages were used to visualize the results. KEGG pathway enrichment based on the Kyoto Encyclopedia of Genes and Genomes (KEGG) database was used to analyze the differences in genes by KOBAS software (KOBAS 3.0) [11], and the statistical test method used was the hypergeometric test. Rich factor refers to the ratio of differentially enriched gene numbers and annotated gene numbers in the pathway. The larger the rich factor is, the greater the degree of enrichment.

qRT-PCR validation of the DEGs from RNA-seq. We performed qRT-PCR of six selected DEGs to confirm the RNA-seq results. The DEG selection criteria are listed as follows: 1) the different changed ratios of DEGs in anaplastic ependymoma tissues and adjacent tissues should be greater than 3 -fold; 2) the FDR value should be less than $0.001 ; 3$ ) the potential genes should be in significantly differently regulated pathways in ependymoma. Quantitative RT-PCR was conducted using an ABI PRISM 7900HT thermocycler (Applied Biosystems, CA). The primers used in qRT-PCR are listed in Table 1. GAPDH was used to normalize the expression data as a reference gene, and the $2^{-\Delta \Delta C t}$ method was applied to calculate the relative expression levels [12].

Statistical analysis. Unpaired Student's t-test was used to examine the significance in qRT-PCR by GraphPad Prism 7 
Table 2. Sample pathological diagnosis information.

\begin{tabular}{|c|c|c|c|c|c|c|c|c|}
\hline Sample & $\begin{array}{c}\text { Age } \\
\text { (months) }\end{array}$ & Pathological diagnosis & $\begin{array}{l}\text { WHO } \\
\text { grade }\end{array}$ & Ki-67 & EMA & GFAP & S-100 & Tumor location \\
\hline Tumor1 & 33 & anaplastic ependymoma & III & $30 \%+$ & - & + & + & right cerebellopontine \\
\hline Tumor2 & 16 & anaplastic ependymoma & III & $10 \%+$ & $+1-$ & + & + & posterior cranial fossa \\
\hline Tumor3 & 11 & anaplastic ependymoma & III & $30 \%+$ & $+1-$ & + & + & right parietal lobe \\
\hline Tumor4 & 47 & anaplastic ependymoma & III & $25 \%+$ & - & + & + & posterior cranial fossa \\
\hline
\end{tabular}

software. Significant differences are marked as ${ }^{*}, * *$, and ${ }^{* * *}$, representing $\mathrm{p}<0.05, \mathrm{p}<0.01$, and $\mathrm{p}<0.001$, respectively.

\section{Results}

Gene expression profiles in anaplastic ependymoma and adjacent tissues. To comprehensively analyze the initiation of anaplastic ependymoma, four anaplastic ependymoma tissues and four corresponding peritumoral tissues were collected for RNA-seq analysis (Table 2). The correlations of the four tumor and adjacent samples ranged from 0.86 to 1 , representing a good correlation between different individual samples. To further verify the similarities and differences of the samples, principal component analysis (PCA) was used to group the samples (Figure 1A). This method grouped the four anaplastic ependymoma samples together and the four adjacent tissues together (Figure 1B). These data indicated the high quality and reliability of the sequencing results. In total, 5,445 differentially expressed genes were identified and selected under strict criteria. A volcano plot was used to show the differentially expressed genes, including 3,042 upregulated and 2,403 downregulated genes (Figure 2A). Hierarchical clustering showed different expression patterns between tumor and adjacent tissues (Figure 2B). The differential genes could not be shown one by one in the thermogram, and they are listed in Supplement material 1.

GO and KEGG pathway enrichment analyses. To gain insights into biological pathways that the upregulated and downregulated differentially expressed genes were involved in, GO and KEGG pathway enrichment analyses were conducted. According to the significance of the enriched GO terms and the number of different genes involved, we chose the top five enriched GO terms for discussion. The top five biological processes of the upregulated differentially expressed genes in the anaplastic ependymoma sample included developmental process, anatomical structure development, multicellular organism development, system development, and animal organ development, while the top five cellular components of the upregulated differentially expressed genes included cytoplasm, cytoplasmic part,

\section{A}

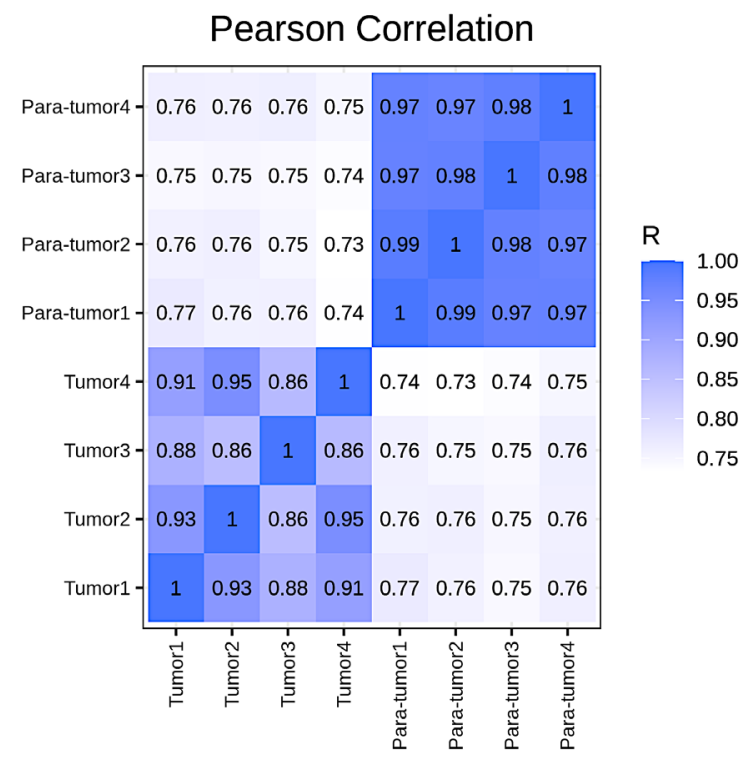

\section{B}

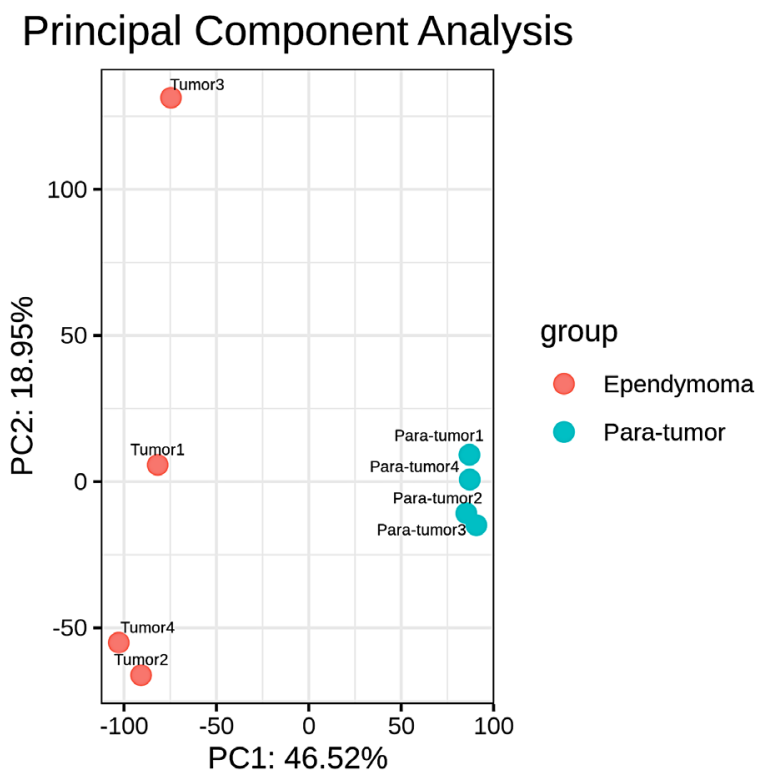

Figure 1. Correlation analysis between tumor samples and paracancer samples. A) Pearson correlation of 4 ependymoma tumor samples and 4 paracancer samples; B) Principal component analysis of 4 ependymoma tumor samples and 4 paracancer samples. 
A

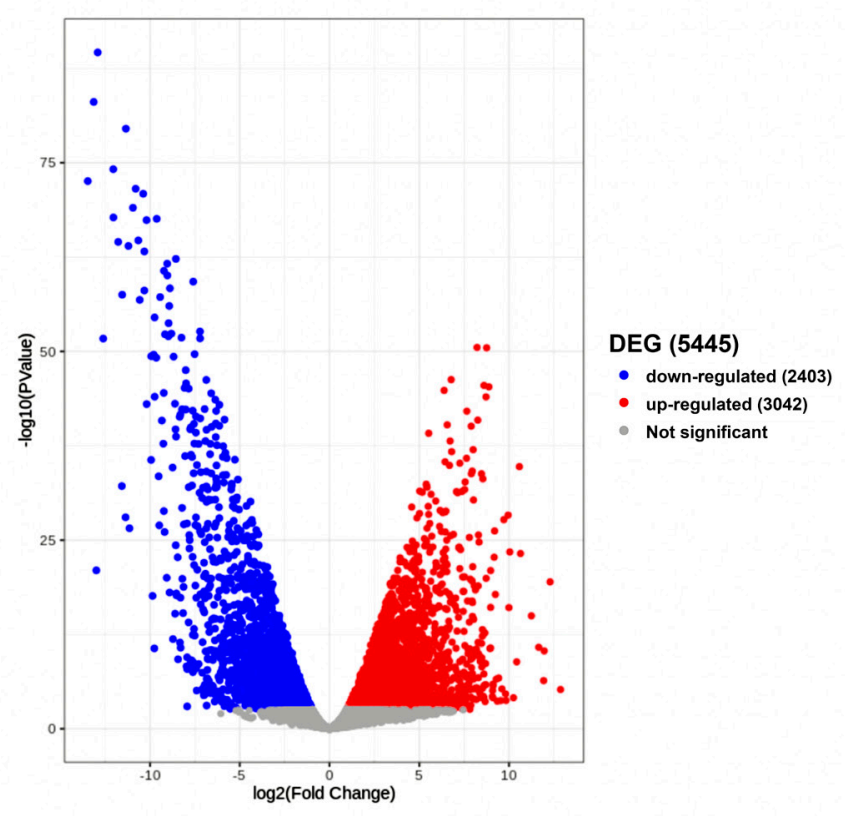

B

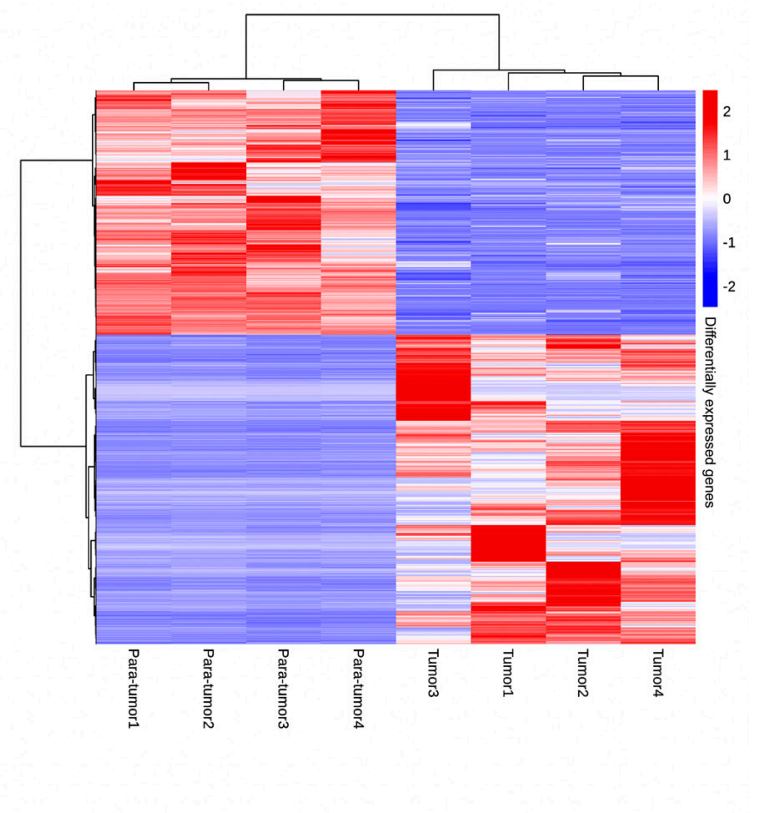

Figure 2. RNA-seq identifies DEGs in ependymoma. A) Volcano plot showed DEGs of 4 pairs of samples based on their p-values and log2 fold change; B) Heat map of the DEGs from 4 pairs of samples. Patient code, condition (tumor, adjacent, or normal), and the relative expression level were shown on the legend.

cytoskeleton, cell projection, and plasma membrane bounded cell projection. Furthermore, protein binding, signaling receptor binding, double-stranded DNA binding, sequencespecific double-stranded DNA binding, and RNA polymerase II regulatory region sequence-specific DNA binding were the top five enriched molecular functions (Figure 3A). For the downregulated differentially expressed genes, nervous system development, regulation of transport, ion transport, cell-cell signaling, and neurogenesis were the five most enriched biological processes. Among the cellular components, cell periphery was the most enriched term, followed by the plasma membrane, plasma membrane part, neuron part, and cell projection. In the molecular function category, the significant terms included transporter activity, transmembrane transporter activity, ion transmembrane transporter activity, cytoskeletal protein binding, and inorganic molecular entity transmembrane transporter activity (Figure 3B). These biological functions with significance in ependymoma were predicted by GSEA, as shown in Figure 4. Moreover, our study showed that 319 KEGG pathways were enriched from adjacent tissues to anaplastic ependymoma tissues, involving all differentially expressed genes. We showed that the upregulated and downregulated differentially expressed genes enriched the 20 significantly regulated signaling pathways with the most significant enrichment degrees between the anaplastic ependymoma and adjacent tissue groups (Figure 5). Among these pathways, ECM-receptor pathway, human papillomavirus infection, focal adhesion,
Hippo signaling pathway, Wnt signaling pathway, proteoglycans in cancer, cell adhesion molecules (CAMs), and MAPK signaling pathway were cancer-related signaling pathways (Supplement material 2).

Quantitative RT-PCR validation. To further verify the RNA-seq results, we used qRT-PCR to verify the mRNA expression of 9 differentially expressed genes, SNAP25, GRM4, CELSR1, LAMA1, WNT5A, ROR2, CCND1, EPHB2, and FOXJ1, in ependymoma and adjacent tissues. The qRT-PCR results confirmed the significant overexpression of CELSR1, LAMA1, WNT5A, ROR2, CCND1, EPHB2, and FOXJ1, and the reduced expression of SNAP25 and GRM4 in ependymoma (Figure 6), suggesting that the RNA-seq data are reproducible and reliable.

\section{Discussion}

Molecular targeted therapy is a therapeutic prescription developed from a deep understanding of tumor etiology, genes, and receptors at the molecular level. Targeted therapeutic drugs could take specific sites of some key molecules in the signal transduction pathway of tumor cells as targets, which can inhibit the growth of tumor cells and promote the inhibition of tumor cell apoptosis by blocking the receptor, inhibiting angiogenesis, and blocking signal transduction to exert stronger antitumor activity, reduce toxicity and side effects on normal cells, and produce a targeted effect on the treatment of tumors [13]. Exploring the molecular targets of 
ependymoma by analyzing mRNAs can be used to establish biomarkers to evaluate the development of ependymoma. In addition, it can also find genes with high activity in the process of tumor development. The findings in this study verify previously reported results. Our study showed that the RELA gene of the principal effector of canonical NF- $\kappa B$ signaling was upregulated, as was C11orf95. Similar findings were also reported in supratypical ependymoma [6]. Both EPHB2 and CCND1, which were found to be overexpressed in this research, were previously validated as ependymoma oncogenes [14-16]. CCND1, a regulator of the cell cycle, is amplified in various cancer types, including breast, head and neck, and bladder cancers [17]. CCND1 is a protein that couples extracellular growth signaling to cell cycle entry through the activation of cyclin-dependent kinase 4 (CDK4) and CDK6 [18]. Upon forming a complex with CCND1, CDK4 and CDK6 phosphorylate and inactivate retinoblastoma (RB), thus leading to the induction of a gene expression program regulated by the E2F family of transcription factors that is important in the transition from G1 to $\mathrm{S}$ phase in the cell cycle [19]. The functions of CCND1 include the control of cell growth, proliferation, transcription, DNA repair, and migration [20]. CCND1 is not essential for cell cycle progression [21]; however, its amplification/overexpression in human tumors is oncogenic, as it allows cancer cells to proliferate independently of extracellular growth signaling cues [17]. Therefore, CCND1 may be an attractive therapeutic target in cancer. The FOXJ1 gene was overexpressed in the anaplastic ependymoma cases in our study and has been reported to have the highest levels in posterior fossa ependymoma group $B$ tumors [22]. The highly expressed genes in this research, such as BOC, PRC1, RFX2, FOSL1, EPHA2, and IGF2BP1, were reported as potential therapeutic molecular targets of ependymoma [23].

Tumor molecular analysis is a basic component of precision oncology that can identify the genomic changes in genes and pathways to achieve therapeutic purposes. There are repeated targeted changes in different histologically defined tumor types coupled with the expanding combination of molecular targeted therapy, which requires flexible and comprehensive methods to analyze clinically significant genes in the whole cancer spectrum [24]. In this study, we also found some genes that may play an important role in anaplastic ependymoma. The most significantly downregulated genes were identified (SNAP25, GRM4, GABRA1, GABRA6, UNC13C, GABRG2, SYNPR, and PVALB) in anaplastic ependymoma. These genes were mostly enriched in the neuron or synapse part in the cellular component category of GO enrichment. According to the KEGG pathway analysis, the synaptic vesicle cycle (hsa04721; p.adjust: 2.1E-12) had the highest rich factor, and 26 out of 50 genes were downregulated. At the same time, the GABAergic synapse pathway and glutamatergic synapse, two synaptic pathways, were enriched in the downregulated genes in ependymoma. This is because synapses are characteristic of mature functional neurons. Moreover, a reduction
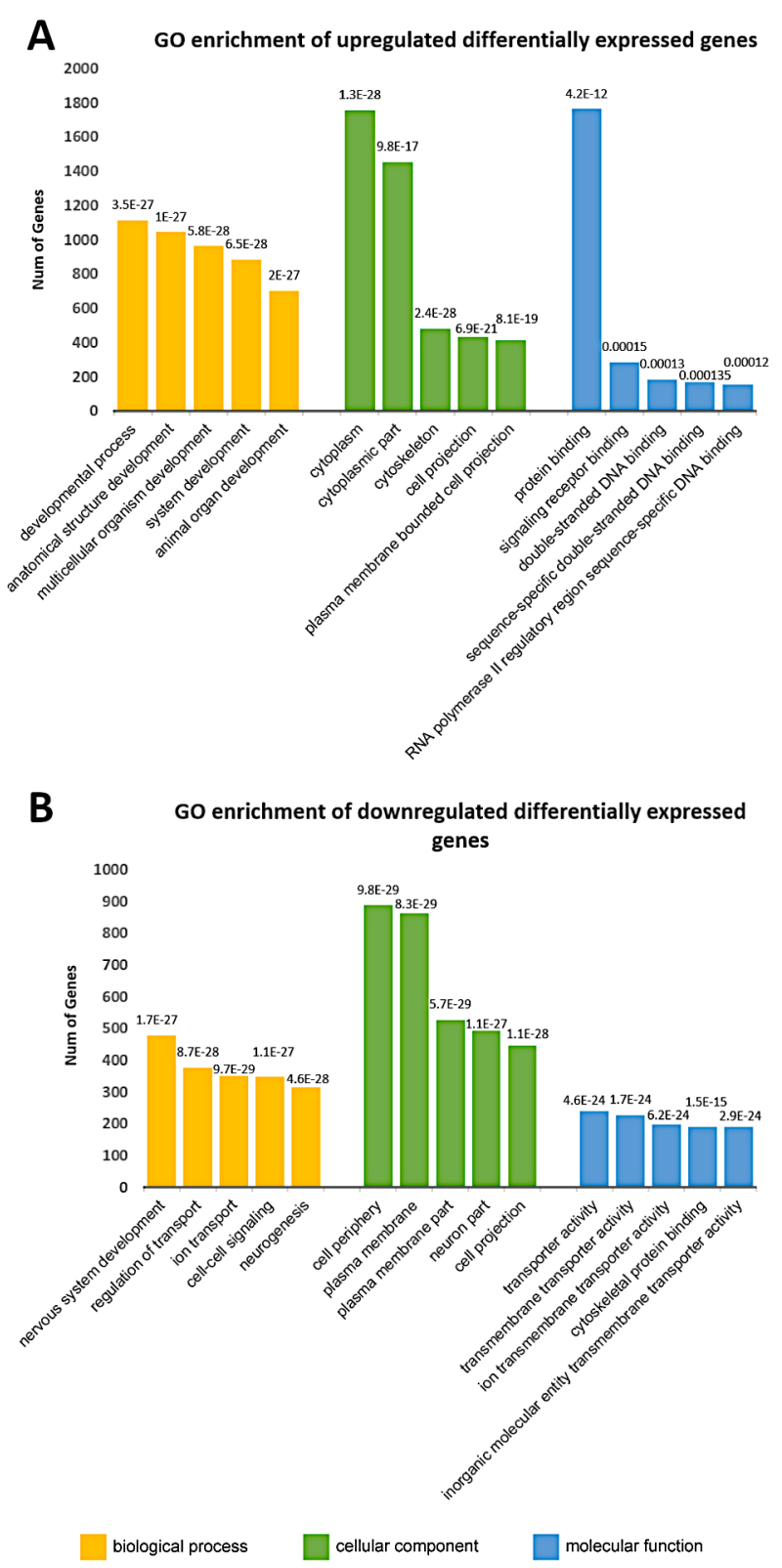

Figure 3. GO enrichment of DEGs. A) Data showed the top five biological processes, cellular component, and molecular functions of upregulated differentially expressed genes in anaplastic ependymoma samples. The false discovery rate (FDR) values are shown on the column chart. B) Data showed the top five biological processes, cellular component, and molecular functions of downregulated differentially expressed genes in anaplastic ependymoma samples.

in synapse-related proteins means that functional neurons may be reduced and normal neuronal signaling transportation could be inhibited. Thus, mature neuronal molecular functions, such as transmembrane transporter activity, ion transmembrane transporter activity, and channel activity, are significantly reduced compared to those in adjacent tumor 

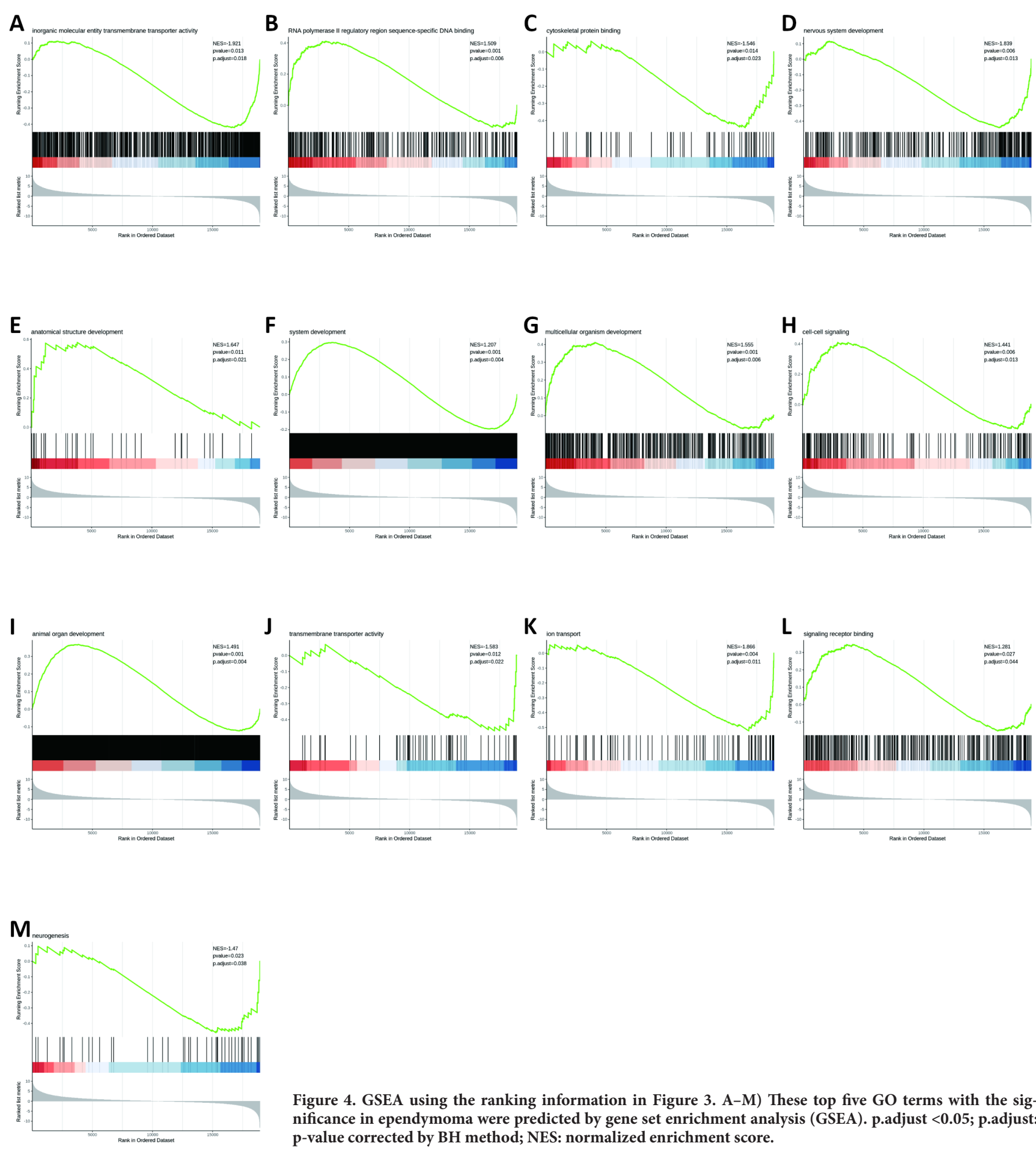

Figure 4. GSEA using the ranking information in Figure 3. A-M) These top five GO terms with the significance in ependymoma were predicted by gene set enrichment analysis (GSEA). p.adjust $<0.05$; p.adjust: p-value corrected by BH method; NES: normalized enrichment score.

tissues. The most significantly upregulated gene, CELSR1, could regulate endothelial adherent junctions and direct cell rearrangements [25]. Upregulated APOH, a commonly methylated gene in cancer, was enriched in protein binding and calcium ion binding [26]. Upregulated VWA3A has been reported to be associated with overall survival and showed enrichment in the extracellular region [27]. It has been reported that ROR2 promotes epithelial-mesenchymal transition by regulating the MAPK/p38 signaling pathways in breast cancer [28]. In this research, overexpressed ROR2 


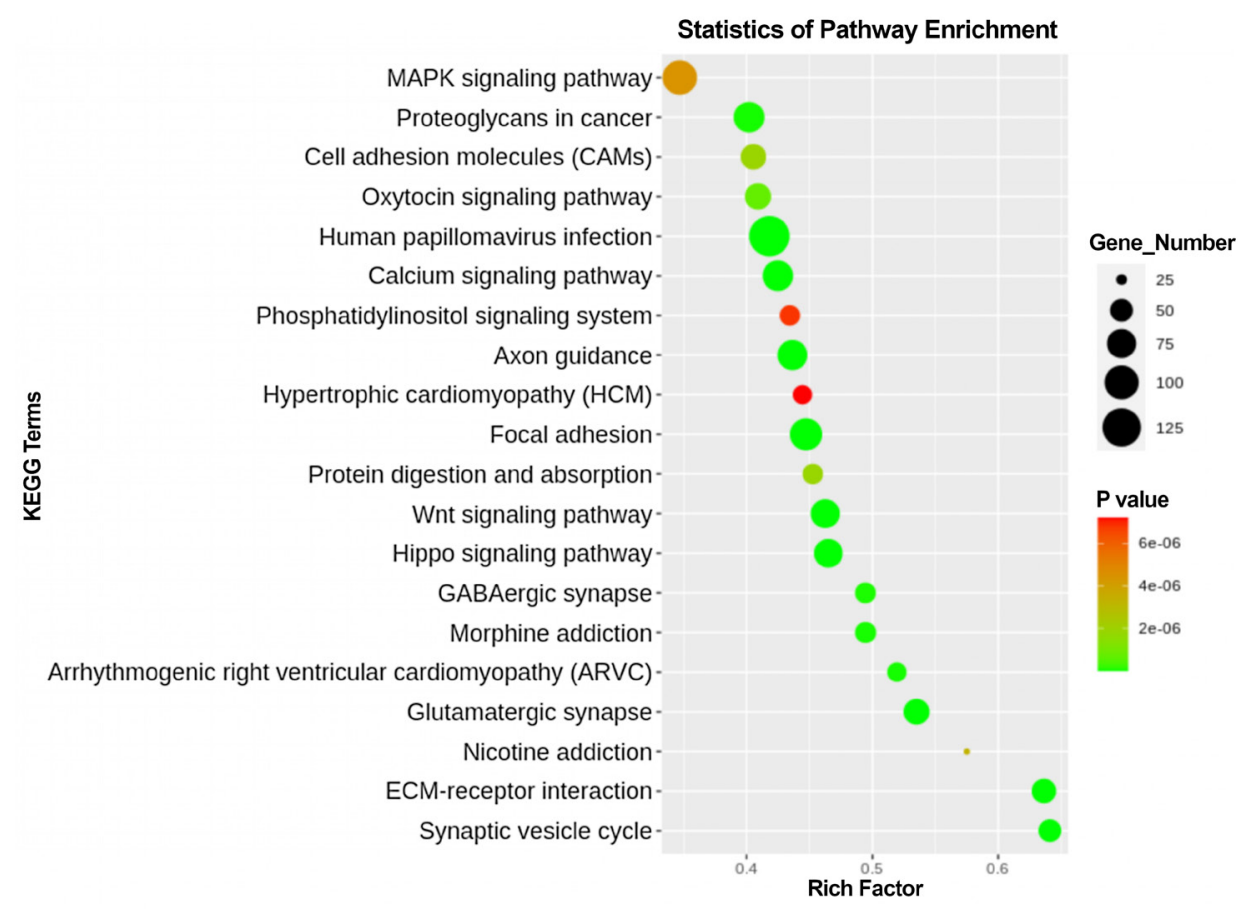

Figure 5. KEGG pathways of the DEGs. Data showed the top 20 significantly regulated signal pathways between anaplastic ependymomas and adjacent groups.
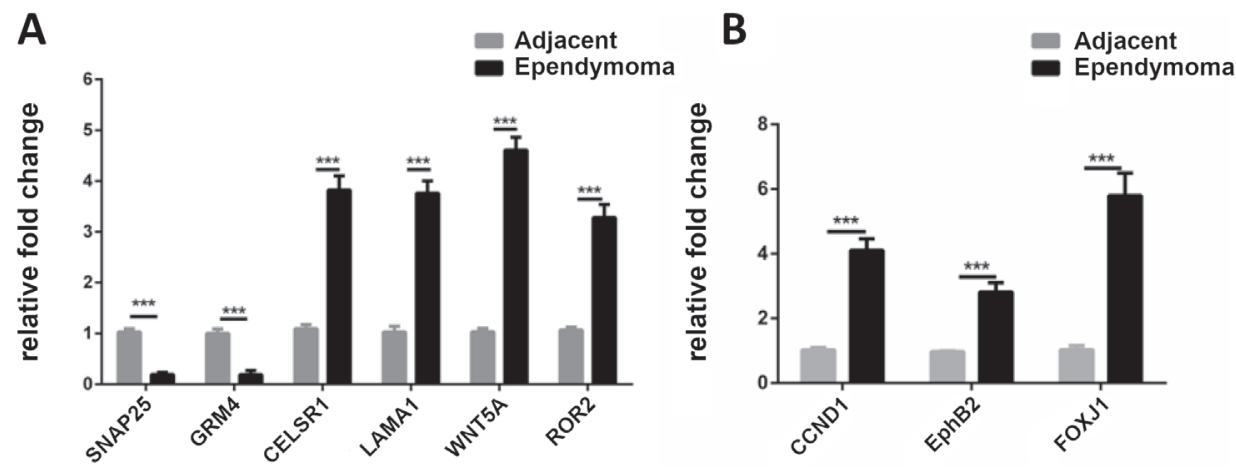

Figure 6. qRT-PCR analysis of SNAP25, GRM4, CELSR1, LAMA1, WNT5A, ROR2, CCND1, EPHB2, and FOXJ1 in ependymoma. Endogenous reference gene GAPDH was used to normalize the average expression of the expression level of DEGs. The logarithm of relative quantitation in the gene expression of corresponding transcripts in 1 tumor tissue/ 1 adjacent tissue. The error bar indicates the standard error of mean \pm SEM. Unpaired Student's T-test was used to examine the significance of expression change by $p$-value $\left.{ }^{\star *} p<0.01 ;{ }^{* * *} p<0.001\right)$.

was enriched in the developmental process and movement of cells or subcellular components. These developmental events included epithelium development, cellular developmental process, and embryo development, which may explain why anaplastic ependymoma has a higher rate of invasion and distant metastasis.

In our results, ECM-receptor was the most significantly enriched pathway (hsa04512, p.adjust: 1.93E-13), with 56 differentially expressed genes out of 80 . It has been reported that the extracellular matrix (ECM) receptor interaction pathway is not only involved in stem cell proliferation but also participates in the adhesion and metastasis of cancers
$[29,30]$. Justin et al. found ECM proteins, including LAMA2, localized to the perivascular GBM niche, which gave rise to a negative prognosis, and they proposed that LAMA2 could serve as a therapeutic target of GBM [31]. Furthermore, LAMA2 was reported to serve as a biomarker of a subgroup of posterior fossa ependymoma with poor prognosis [32]. In this study, we found upregulated mRNA levels of three major ECM receptor proteins, laminin (LAMA1, LAMA2, LAMA3) and collagen (COL6A2, COL5A2, COL4A5, COL3A1, COL2A1). These genes were significantly upregulated in 4 anaplastic ependymoma tissues, which indicates that laminin plays an important role in anaplastic ependy- 
moma and that the ECM receptor interaction pathway is activated in ependymoma. Therefore, laminin (LAMA1) and collagen (COL2A1) may act as ependymoma biomarkers, and the inhibition of the ECM receptor interaction pathway may have a significant effect on ependymoma therapy.

Moreover, WNT11, WNT5A, and WNT1 were upregulated in the anaplastic ependymoma group and enriched in many pathways, such as the Hippo signaling pathway, Wnt signaling pathway, and proteoglycans in cancer. It has been reported that overexpressed WNT1 expression is associated with glioma [33] and metastasis in cholangiocarcinoma [34]. WNT5A was reported to be produced by the choroid plexus of the developing hindbrain and plays an essential role in regulating developmental pathways such as hindbrain morphogenesis [35]. WNT5A is also a protooncogene and enhances the migration of chronic lymphocytic leukemia cells $[36,37]$. Wnt family proteins are secreted and can be transported via lipoprotein particles [35]. Therefore, overexpressed WNT1, WNT11, and WNT5A may be secreted to enhance cell-cell migration and lead to tumors. We propose that they may play an important role in the pathogenesis of ependymoma, and their functions in ependymoma tumorigenesis need to be further investigated.

Overall, we obtained a profile of differential gene expression from clinical anaplastic ependymoma tissues. Through the analysis and discussion of the profiling results, we selected 9 differentially expressed genes between anaplastic ependymoma tissues and adjacent tumor tissues for validation by RT-qPCR (SNAP25, GRM4, CELSR1, LAMA1, WNT5A, ROR2, CCND1, EPHB2, and FOXJ1). Correspondingly, the synaptic vesicle cycle pathway and extracellular matrix (ECM) receptor interaction pathway were highly enriched in the anaplastic ependymoma group, possibly providing a reference for the clinical understanding of ependymoma, which improved our understanding of the carcinogenesis of anaplastic ependymoma. This study provides global gene information and some new molecular targets or candidate biomarkers for ependymoma diagnosis and therapy.

Supplementary information is available in the online version of the paper.

Acknowledgments: This work was supported by the Science and Technology Research and Development Fund of Shenzhen science and technology innovation commission (JCYJ20160429174426094). The funders had no role in the study design, data collection and analysis, decision to publish, or the manuscript preparation.

\section{References}

[1] MERCHANT TE, LI C, XIONG X, KUN LE, BOOP FA et al. Conformal radiotherapy after surgery for paediatric ependymoma: a prospective study. Lancet Oncol 2009; 10: 258-266. https://doi.org/10.1016/s1470-2045(08)70342-5
[2] LOUIS DN, PERRY A, REIFENBERGER G, VON DEIMLING A, FIGARELLA-BRANGER D et al. The 2016 World Health Organization Classification of Tumors of the Central Nervous System: a summary. Acta Neuropathol 2016; 131: 803-820. https://doi.org/10.1007/s00401-016-1545-1

[3] KHATUA S, MANGUM R, BERTRAND KC, ZAKY W, MCCALL D et al. Pediatric ependymoma: current treatment and newer therapeutic insights. Future Oncol 2018; 14: 3175-3186. https://doi.org/10.2217/fon-2018-0502

[4] GERSTNERER, PAJTLER KW. Ependymoma. Semin Neurol 2018; 38: 104-111. https://doi.org/10.1055/s-0038-1636503

[5] LUKASHOVA-V ZANGEN I, KNEITZ S, MONORANU CM, RUTKOWSKI S, HINKES B et al. Ependymoma gene expression profiles associated with histological subtype, proliferation, and patient survival. Acta Neuropathol 2007; 113: 325-337. https://doi.org/10.1007/s00401-006-0190-5

[6] PARKER M, MOHANKUMAR KM, PUNCHIHEWA C, WEINLICH R, DALTON JD et al. C11orf95-RELA fusions drive oncogenic NF-kappaB signalling in ependymoma. Nature 2014; 506: 451-455. https://doi.org/10.1038/nature13109

[7] XIAO CL, MAI ZB, LIAN XL, ZHONG JY, JIN JJ et al. FANSe2: A Robust and Cost-Efficient Alignment Tool for Quantitative Next-Generation Sequencing Applications. PLoS One 2014; 9: e94250. https://doi.org/10.1371/journal. pone.0094250

[8] BLOOM JS, KHAN Z, KRUGLYAK L, SINGH M, CAUDY AA. Measuring differential gene expression by short read sequencing: quantitative comparison to 2-channel gene expression microarrays. BMC Genomics 2009; 10: 221. https:// doi.org/10.1186/1471-2164-10-221

[9] ROBINSON MD, MCCARTHY DJ, SMYTH GK. edgeR: a Bioconductor package for differential expression analysis of digital gene expression data. Bioinformatics 2010; 26: 139140. https://doi.org/10.1093/bioinformatics/btp616

[10] RAJKUMAR AP, QVIST P, LAZARUS R, LESCAI F, JU J et al. Experimental validation of methods for differential gene expression analysis and sample pooling in RNA-seq. BMC Genomics 2015, 16:548. BMC Genomics 2015; 16: 548. https://doi.org/10.1186/s12864-015-1767-y

[11] XIE C, MAO X, HUANG J, DING Y, WU J et al. KOBAS 2.0: a web server for annotation and identification of enriched pathways and diseases. Nucleic Acids Res 2011; 39: W316322. https://doi.org/10.1093/nar/gkr483

[12] SCHEFE JH, LEHMANN KE, BUSCHMANN IR, UNGER T, FUNKE-KAISER H. Quantitative real-time RT-PCR data analysis: current concepts and the novel "gene expression's CTdifference” formula. J Mol Med (Berl) 2006; 84: 901-910. https://doi.org/10.1007/s00109-006-0097-6

[13] JOHNSON RA, WRIGHT KD, POPPLETON H, MOHANKUMAR KM, FINKELSTEIN D et al. Cross-species genomics matches driver mutations and cell compartments to model ependymoma. Nature 2010; 466: 632-636. https://doi. org/10.1038/nature09173

[14] MOHANKUMAR KM, CURRLE DS, WHITE E, BOULOS $\mathrm{N}$, DAPPER J et al. An in vivo screen identifies ependymoma oncogenes and tumor-suppressor genes. Nat Genet 2015; 47: 878-887. https://doi.org/10.1038/ng.3323 
[15] TAYLOR MD, POPPLETON H, FULLER C, SU X, LIU Y et al. Radial glia cells are candidate stem cells of ependymoma. Cancer Cell 2005; 8: 323-335. https://doi.org/10.1016/j. ccr.2005.09.001

[16] ABEDALTHAGAFI MS, WU MP, MERRILL PH, DU Z, WOO $\mathrm{T}$ et al. Decreased FOXJ1 expression and its ciliogenesis programme in aggressive ependymoma and choroid plexus tumours. J Pathol 2016; 238: 584-597. https://doi. org/10.1002/path.4682

[17] MACK SC, PAJTLER KW, CHAVEZ L, OKONECHNIKOV $\mathrm{K}$, BERTRAND KC et al. Therapeutic targeting of ependymoma as informed by oncogenic enhancer profiling. Nature 2018; 553: 101-105. https://doi.org/10.1038/nature25169

[18] TATIN F, TADDEI A, WESTON A, FUCHS E, DEVENPORT D et al. Planar cell polarity protein Celsr1 regulates endothelial adherens junctions and directed cell rearrangements during valve morphogenesis. Dev Cell 2013; 26: 3144. https://doi.org/10.1016/j.devcel.2013.05.015

[19] OHARA K, ARAI E, TAKAHASHI Y, ITO N, SHIBUYA A et al. Genes involved in development and differentiation are commonly methylated in cancers derived from multiple organs: a single-institutional methylome analysis using 1007 tissue specimens. Carcinogenesis 2017; 38: 241-251. https:// doi.org/10.1093/carcin/bgw209

[20] MADDEN SF, CLARKE C, STORDAL B, CAREY MS, BROADDUS R et al. OvMark: a user-friendly system for the identification of prognostic biomarkers in publically available ovarian cancer gene expression datasets. Mol Cancer 2014; 13: 241. https://doi.org/10.1186/1476-4598-13-241

[21] XU J, SHI J, TANG W, JIANG P, GUO M et al. ROR2 promotes the epithelial-mesenchymal transition by regulating MAPK/p38 signaling pathway in breast cancer. J Cell Biochem 2020; 121: 4142-4153. https://doi.org/10.1002/ jcb.29666

[22] YEH MH, TZENG YJ, FU TY, YOU JJ, CHANG HT et al. Extracellular Matrix-receptor Interaction Signaling Genes Associated with Inferior Breast Cancer Survival. Anticancer Res 2018; 38: 4593-4605. https://doi.org/10.21873/anticanres. 12764
[23] BAO Y, WANG L, SHI L, YUN F, LIU X et al. Transcriptome profiling revealed multiple genes and ECM-receptor interaction pathways that may be associated with breast cancer. Cell Mol Biol Lett 2019;24: 38. https://doi.org/10.1186/s11658019-0162-0

[24] LATHIA JD, LI M, HALL PE, GALLAGHER J, HALE JS et al. Laminin alpha 2 enables glioblastoma stem cell growth. Ann Neurol 2012; 72: 766-778. https://doi.org/10.1002/ ana. 23674

[25] WITT H, MACK SC, RYZHOVA M, BENDER S, SILL M et al. Delineation of two clinically and molecularly distinct subgroups of posterior fossa ependymoma. Cancer Cell 2011; 20: 143-157. https://doi.org/10.1016/j.ccr.2011.07.007

[26] YU X, WANG M, WU J, HAN Q, ZHANG X. ZNF326 promotes malignant phenotype of glioma by up-regulating HDAC7 expression and activating Wnt pathway. J Exp Clin Cancer Res 2019; 38: 40. https://doi.org/10.1186/s13046019-1031-4

[27] ZHAO W, YANG S, CHEN J, ZHAO J, DONG J. Forced overexpression of FBP1 inhibits proliferation and metastasis in cholangiocarcinoma cells via Wnt/beta-catenin pathway. Life Sci 2018; 210: 224-234. https://doi.org/10.1016/j. lfs.2018.09.009

[28] KAISER K, GYLLBORG D, PROCHAZKA J, SALASOVA A, KOMPANIKOVA P et al. WNT5A is transported via lipoprotein particles in the cerebrospinal fluid to regulate hindbrain morphogenesis. Nat Commun 2019; 10: 1498. https:// doi.org/10.1038/s41467-019-09298-4

[29] CLARK CC, COHEN I, EICHSTETTER I, CANNIZZARO LA, MCPHERSON JD et al. Molecular cloning of the human proto-oncogene Wnt-5A and mapping of the gene (WNT5A) to chromosome 3p14-p21. Genomics 1993; 18: 249-260. https://doi.org/10.1006/geno.1993.1463

[30] HASAN MK, RASSENTI L, WIDHOPF GF, 2ND, YU J, KIPPS TJ. Wnt5a causes ROR1 to complex and activate cortactin to enhance migration of chronic lymphocytic leukemia cells. Leukemia 2019; 33: 653-661. https://doi. org/10.1038/s41375-018-0306-7 